\title{
Nonlinear Correlation Model in the Assessment of Screen Printing Indoor Pollution
}

\author{
Jelena Kiurski ${ }^{*}$, Ljubo Nedović, Savka Adamović, Ivana Oros, Jelena Krstić, Ilija Kovačević, \\ Lidija Čomić
}

University of Novi Sad, Faculty of Technical Sciences, Trg Dositeja Obradovića 6, 21000 Novi Sad, Serbia

\begin{abstract}
The correlation models were applied to assess the mutual dependencies of the ozone concentrations on the sampling time and sampling points as an extension of our previous investigations. There is no significant mutual dependence, but there are some observable linear and non-linear correlations with sampling time. Correlation of ozone concentration on sampling time is linear at two sampling points ( 6 and 5) and is expressed by equation: $y=-0.0171 x+0.1329$ for sampling point 6 . At the other sampling points $\left(1\right.$ and 3 ) nonlinear correlations are expressed by third degree polynomials, $y=0.0061 x^{3}$ $-0.0934 x^{2}+0.4335 x+0.0967$ (sampling point 1$), y=0.0035 x^{3}-0.0520 x^{2}+0.2182 x+0.9887$ (sampling point 3 ). All calculations have been done in the program MATHEMATICA, version 7.0, at 4 correct decimals. Nonlinear correlation model can be successfully used in the assessment of indoor polluted gases distribution in screen printing facility.
\end{abstract}

Keywords Regression models, Ozone, Monitoring, Screen printing, Indoor pollution

\section{Introduction}

Screen printing is arguably the most versatile of all printing techniques. It can be used to print on a wide variety of substrates, including paper, paperboard, plastics, glass, metals, fabrics, and many other materials. Because of the simplicity of the application, a wider range of inks and dyes are available in screen printing than in other printing techniques. This has been a result of the development of the automated and rotary screen printing press, improved driers, and UV curable ink [1,2]. UV curable inks consist of liquid prepolymers, monomers, and initiators which upon being exposed to large doses of UV radiation instantly polymerize the vehicle to a dry, tough thermosetting resin [1-4]. Ozone is produced incidentally during many printing activities where UV radiation is emitted, during curing of printing inks, varnishes, lacquers, use of projection lamps, etc. [5]. The risks to health are slight - and in general any symptoms shown will be irritation of the respiratory tract [6-8]. However, in certain cases it is possible that the level of ozone accumulated in inadequately ventilated rooms exceeds the Occupational Exposure Standard (OES). The OESs are approved by the Health and Safety Commission and are applicable only to persons at work. The current OES for ozone is $0.2 \mathrm{ppm}$ in air, averaged over a 15 minute reference pe$\operatorname{riod}[9]$.

* Corresponding author:

kiurski@uns.ac.rs (Jelena Kiurski)

Published online at http://journal.sapub.org/ajee

Copyright (C) 2012 Scientific \& Academic Publishing. All Rights Reserved
The previous studies were focused mainly on the measurements of tropospheric ozone and other pollutants and their statistical analysis [10-15]. The mutual dependence of ozone concentration on the sampling points and sampling time in indoor printing environment has not been examined yet.

This paper extends on the results of our previous investigation for detection and quantification of ozone in the screen printing facilities of Novi Sad [16]. Also, this paper describes the development of an application to forecast the peak ozone levels with the aid of air quality variables, in screen printing facilities. For this purpose, a regression model was considered. The model adapted includes variables that are available on a daily basis, so as daily operational maximum ozone concentration level forecast can be achieved.

\section{Materials and Methods}

Sampling: Concentration levels of ozone were monitored in two small screen printing facilities (4-12 employees) in Novi Sad, situated on northern part of Serbia. The process in facility 1 (SPF 1) is automatic with a significantly higher production compared with manual process in facility 2 (SPF 2).

Number of sampling points during instrumental measurement of ozone concentration was different, due to the type of production process (five sample points in automatic and one in manual process). The locations of sampling points during instrumental measurement were determined by the technical characteristics of automatic screen printing ma- 
chine, Table 1 [16].

During analytical measurement method, air was sampled at one sampling point, near to the automatic machine and to the desk for manual screen printing process.

Table 1. Location of Sampling Points During Instrumental Measurement

\begin{tabular}{cl}
\hline $\begin{array}{c}\text { Sampling } \\
\text { point }\end{array}$ & \multicolumn{1}{c}{ Description } \\
\hline 1 & Fitting of sheets \\
2 & Printing process \\
3 & Entrance to the UV drying unit \\
4 & Exit from the UV drying unit \\
5 & Delivery of sheets \\
6 & Desk for manual screen printing process \\
\hline
\end{tabular}

Instrumental method: The detection and quantification of ozone was performed in situ during working time ( 8 hours) by ozonometer, Aeroqual Series 200, Aeroqual Ltd.

Analytical method: The air was collected during 24 hours by air sampler PRO-EKOS AT. 401X and infiltrated through the gas washing bottles (acc. to Drechsel) with filter disks containing absorption solution for ozone. Air flow was 0.2 to $0.4 \mathrm{dm}^{3} \mathrm{~min}^{-1}$. The ozone concentrations were determined by spectrophotometric method (UV/VIS spectrophotometer DR 5000 HACH LANGE) at $352 \mathrm{~nm}[16,17]$.

Reagents: All the chemicals were of analytical reagent grade (Merck, Germany).

Statistical Analysis: For the purposes of statistical analysis the linear and nonlinear regression were applied in order to determine the mutual dependencies of the ozone concentrations on the sampling time and sampling points. Since ozone is mostly generated in the vicinity of UV lamps, the application of linear and nonlinear regression could determine how the ozone spreads within the screen printing indoor, and how it affects the employees depending on their distance from the critical areas. Also, the application of regression could allow the prediction of time when the employees were the most exposed to ozone and when they need to be sufficiently far from the critical place.

\section{Results and Discussion}

Table 2. Ozone Concentrations During $8 \mathrm{~h}$ Measurements, Instrumental Method [16]

\begin{tabular}{cccccccc}
\hline \multirow{2}{*}{$\begin{array}{c}\text { Sampling } \\
\text { time (h) }\end{array}$} & \multicolumn{7}{c}{ Ozone concentrations (ppm) } \\
\cline { 2 - 8 } & Sampling facility & \multicolumn{5}{c}{ SPF 1 } & SPF 2 \\
\cline { 2 - 8 } & Sampling point & 1 & 2 & 3 & 4 & 5 & 6 \\
\hline 1 & & 0.436 & 0.647 & 0.812 & 0.213 & 0.812 & 0.118 \\
2 & & 0.647 & 0.541 & 0.754 & 0.775 & 0.803 & 0.085 \\
3 & & 0.738 & 0.712 & 0.695 & 1.041 & 0.794 & 0.080 \\
4 & & 0.712 & 0.695 & 0.712 & 1.332 & 0.000 & 0.090 \\
5 & & 0.689 & 0.738 & 0.775 & 2.228 & 0.000 & 0.058 \\
6 & & 0.647 & 0.436 & 0.812 & 8.132 & 0.000 & 0.000 \\
7 & & 0.689 & 0.647 & 0.803 & 8.503 & 0.000 & 0.018 \\
8 & & 0.712 & 0.794 & 0.794 & 0.000 & 0.000 & 0.000 \\
\hline
\end{tabular}

Table 3. Ozone Concentrations During $8 \mathrm{~h}$ (Instrumental) and 24h (Analytical) Measurements [16]

\begin{tabular}{cccccccc}
\hline \multirow{2}{*}{$\begin{array}{c}\text { Sampling } \\
\text { time (h) }\end{array}$} & \multicolumn{7}{c}{ Ozone concentrations (ppm) } \\
\cline { 2 - 8 } & Sampling facility & SPF 1 & \multicolumn{7}{c}{ SPF 2 } \\
\hline 8 & & 1 & 2 & 3 & 4 & 5 & 6 \\
\hline 24 & & 0.659 & 0.651 & 0.770 & 2.778 & 0.301 & 0.056 \\
& & & & 1.028 & & & 0.299 \\
\hline
\end{tabular}


Table 5. Dependence of Correlation Coefficients of Ozone Concentrations on the Sampling Time at the Observed Sampling Points

\begin{tabular}{cccccc}
\hline 1 & 2 & 3 & 4 & 5 & 6 \\
0.5644 & 0.1968 & 0.3419 & 0.4959 & -0.8476 & -0.9299 \\
\hline 1 - 5 - sampling points in screen printing facility 1 \\
6 - sampling point in screen printing facility 2 \\
\hline
\end{tabular}

The graph of linear regression dependence of ozone concentration on sampling time at sampling point 6 is presented in Figure 1. The corresponding correlation coefficient is -0.9299 , and the equation of regression line is: $y=-0.0171 x+0.1329$ (all data obtained in MATHEMATICA, at 4 correct decimals).

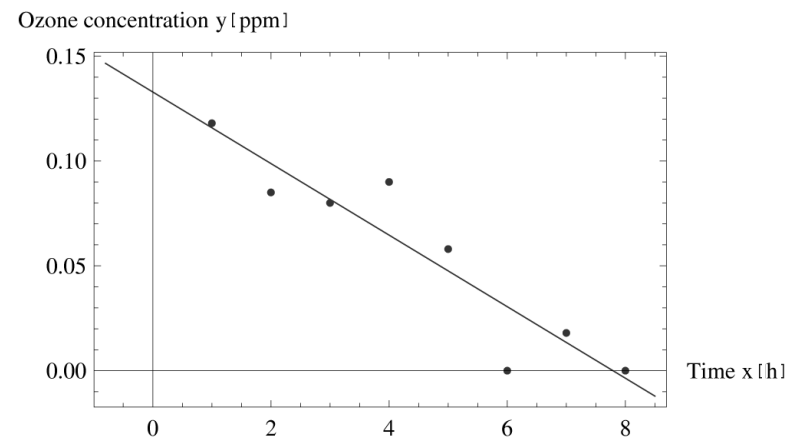

Figure 1. Graph of linear regression at sampling point 6 , screen printing facility 2

Nonlinear dependence of ozone concentration on sampling time: Investigating a possible nonlinear dependence of ozone concentration on sampling time at observed sampling points, it was observed that the sampling points 1 and 3 (automatic printing) can be modelled with polynomial equation of third degree (all data obtained in MATHEMATICA, at 4 correct decimals).

Graph of nonlinear regression of third degree on sampling point 1, screen printing facility 1 , Figure 2, presents the dependence of ozone concentration on sampling time. The equation of this curve is: $y=0.0061 x^{3}-0.0934 x^{2}+0.4335 x+$ 0.0967 .

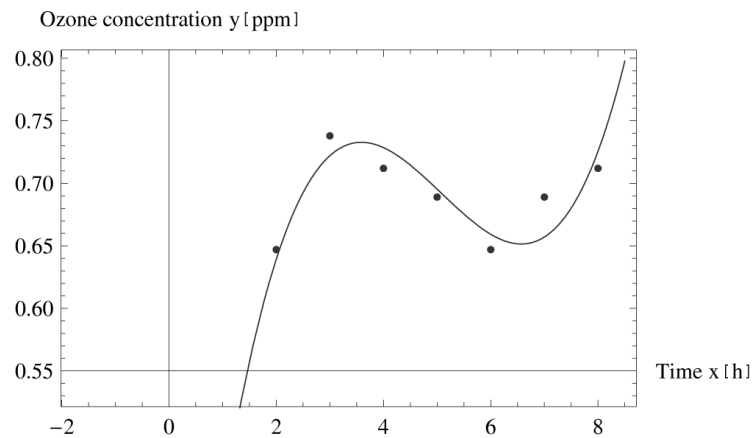

Figure 2. Graph of nonlinear regression of third degree at sampling point 1 , screen printing facility 1

Figure 3 presents a graph of the regression curve of third degree of the ozone concentrations dependence on sampling time at sampling point 3 . The equation of this curve is: $y=0.0035 x^{3}-0.0520 x^{2}+0.2182 x+0.9887$.

According to the number of samples (8), third-polynomial approximation is still very approximate, and some other functional dependence at other sampling points is not obvi- ous.

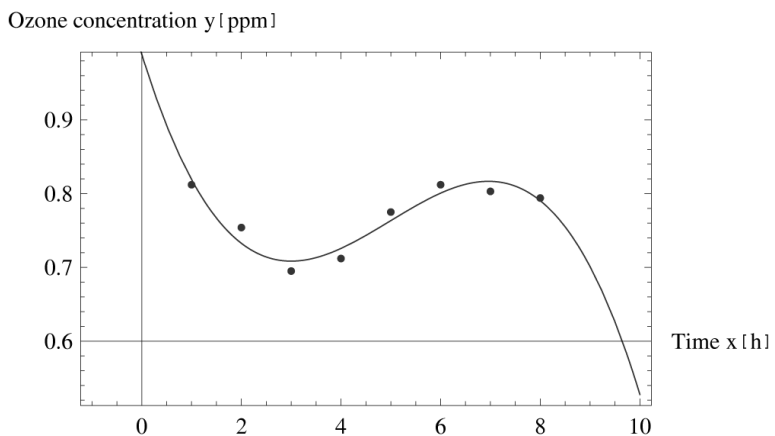

Figure 3. Graph of nonlinear regression of third degree at sampling point 3 , screen printing facility 1

Applying linear and nonlinear correlation models on the obtained data, it was concluded that:

- Distance between sampling points has low effect on the concentration of ozone;

- Concentration of ozone decreases during the 8 hours production process;

- Based on the nonlinear correlation model the place and time point of the maximum ozone concentration in the screen printing facilities can be determined.

Correlation of ozone concentration on sampling time is linear at two sampling points (6 and 5) and is expressed by equation: $y=-0.0171 x+0.1329$ for sampling point 6 . At the other sampling points (1 and 3 ) nonlinear correlations are expressed by third degree polynomials,

$y=0.0061 x^{3}-0.0934 x^{2}+0.4335 x+0.0967$ (sampling point 1),

$y=0.0035 x^{3}-0.0520 x^{2}+0.2182 x+0.9887$ (sampling point 3).

Practical application of the derived equations is to determine time points of the maximum ozone concentrations during the screen printing process. At sampling points 1 and 3 (SPF 1), time points of the maximum ozone concentrations are 3 and 7 hours, respectively.

\section{Conclusions}

Our previous study clearly showed that concentration of ozone depends on the type of screen printing process (automatic or manual) and increases with production volume. Because of the short half-life of ozone (15 minutes) and its known interaction with other pollutants (VOC, $\mathrm{NO}, \mathrm{NO}_{2}$, PAN) present in indoor of screen printing facilities, detected concentrations are probably lower than real generated concentrations. Namely, the ozone concentration has a local character, meaning that it can be different at physically close locations, and can be independent of the ozone concentrations at nearby locations. Thus, we conclude that ozone is quickly eliminated in the contact with the surrounding air and is removed outdoors through ventilation systems.

Considering the printing microclimate and the complexity of air quality determination, the quantity of actual measured data needed to produce a far reaching and reliable prediction 
leads very fast to a problem difficult to be managed. The statistical linear regression analysis model becomes, in that sense, attractive, as it enables a fast assessment of the location-specific situation. Even so, given the inevitable limits of regression analysis, the use of area and time covering monitoring data remains a prerequisite. It can, therefore, be only emphasised, that the statistical methodology should be guided by an understanding of the underlying physical mechanisms.

Therefore, this study could be a starting point in application of linear and nonlinear regression for ozone analysis in other types of press processes.

\section{ACKNOWLEDGEMENTS}

The authors acknowledge the financial support of the Ministry of Education and Science of the Republic of Serbia, within the Project No. 34014.

\section{REFERENCES}

[1] Hancock, A., Lin, L., 2004, Challenges of UV curable ink-jet printing inks - a formulator's perspective, Pigment and Resin Technology, 33(5), 280-286.

[2] Yoichi, I., 2003, Functional Screen Printing Technologies. Present and Future. Present Status and Future of Functional Screen Printing Technology, Bulletin of the Japanese Society of Printing Science and Technology, 40(1), 2-9.

[3] Printers' National Environmental Assistance Center (PNEAC). (1995) Print Process Descriptions: Printing Industry Overview: Screen Printing. [Online]. Available: http://www.pneac.org/printprocesses/screen/

[4] Occupational Safety and Health Branch Labour Department. (2004) Chemical Safety in the Workplace. Guidance Notes on Chemical Safety in Printing Industry. [Online]. Available: http://www.labour.gov.hk/eng/public/os/C/GN_Printing.pdf

[5] Canadian Council of Ministers of the Environment. (2003) Effect of ozone on materials. [Online]. Available: http://www.ccme.ca/assets/pdf/screvw_oz_effects_materials e.pdf
[6] Jakobi, G., and Fabian, P., 1997, Indoor/outdoor concentrations of ozone and peroxyacetylnitrate (PAN), International Journal of Biometeorology, 40(3), 162-165.

[7] Curtis, L., Rea, W., Smith-Willis, P., Fenyves, E., and Pan, Y., 2006, Adverse health effects of outdoor air pollutants, Environment International, 32, 815-830.

[8] Wolkoff, P., Clausen, P. A., Wilkins, C. K., and Nielsen, G. D., 2000, Formation of strong airway irritants in terpene/ozone mixtures, Indoor Air, 10, 82-91.

[9] Health and Safety Department, the University of Edinburgh. (2010) Photocopiers and laser printers health hazards. [Online]. Available: http://www.docs.csg.ed.ac.uk/ photocopiers.pdf

[10] Zolghadri, A., Monsion, M., Henry, D., Marchionini, C., and Petrique, O., 2004, Development of an operational model-based warning system for tropospheric ozone concentrations in Bordeaux, France, Environmental Modelling \& Software, 19(4), 369-382.

[11] G. A. F. Seber, and C. J. Wild, Nonlinear Regression, New York: John Wiley and Sons, 1989.

[12] R. M. Bethea, B. S. Duran, and T. L. Boullion, Statistical Methods for Engineers and Scientists, New York: Marcel Dekker, 1985.

[13] Thompson, M. L., Reynolds, J., Cox, L. H., Guttorp, P., and Sampson, P., 2001, A review of statistical methods for the meteorological adjustment of tropospheric ozone, Atmospheric Environment, 35(3), 617-630.

[14] Statistics Canada. (2005) Canadian Environmental Sustainability Indicators: Air Quality Indicator: Data Sources and Methods. (Catalogue no. 16-254-XIE)

[15] Aceves-Fernandez, M. A., Ramos-Arreguín, J. M., Pedraza-Ortega, J. C., Sotomayor-Olmedo, A., and Tovar-Arriaga, S., 2011, Finding trends of airborne harmful pollutants by using recurrence quantification analysis, American Journal of Environmental Engineering, 1(1), 10-14.

[16] Kiurski, J., Adamović, S., Oros, I., Krstić, J., and Djogo, M., 2011, Detection and quantification of ozone in screen printing facilities, International Journal of Civil and Environment Engineering, 3(3), 137-142.

[17] National Institute for Occupational Safety and Health (NIOSH), Standard method for ozone determination, Method No: P \&CAM 154, 2002. 